\title{
Abell 1763 and its Cluster Feeding Filament: Constraints on the Intra-filament Medium
}

\author{
Louise O. V. Edwards ${ }^{1}$ and Dario Fadda ${ }^{1}$ \\ ${ }^{1}$ California Institute of Technology/Herschel Science Center \\ 1200 E. California Blvd. Pasadena, CA, 91125 \\ email: louise@ipac.caltech.edu
}

\begin{abstract}
Galaxies are found to inhabit a variety of environments. They are often found in pairs, groups, or clusters. Cosmological simulations predict that these clusters are connected on a larger scale by filaments, but because these large scale structures are so vast, and because they are of intermediate density, observational constraints on their properties are difficult to achieve. We find a large-scale filament in the Abell 1763-Abell 1770 superstructure, determine that the star-formation therein is enhanced, and uncover a bent double lobe radio source midway across the filament. From the bend of this AGN'a jets, we probe the density of the surrounding intra-filament medium (IFM), constrained to be between $1-20 \times 10^{-29} \mathrm{gm} / \mathrm{cm}^{3}$. This density is consistent with direct probes of the IFM as well as theoretical models.
\end{abstract}

Keywords. galaxies: active, galaxies:evolution, cosmology:large-scale structure of the universe, X-rays: galaxies: clusters

\section{Introduction}

Connected by vast filaments, and separated by huge voids, galaxy clusters are the largest bound systems in our universe. The galaxies which inhabit clusters are bathed in a hot $\left(\mathrm{T} \sim 10^{8} \mathrm{~K}\right)$, dense $\left(\mathrm{n}_{e} \sim 10^{-3} \mathrm{~cm}^{-3}\right)$ intra-cluster medium which permeates throughout the structure. Although these galaxies are often thought of as 'old, red, and dead' there are regions within the structure where the galaxies are active, not only in the radio, but also in the infrared and in optical emission lines.

As a galaxy enters the cluster virial radius, interactions with the hot intra-cluster medium or neighbouring galaxies can compress the interstellar medium, invigorating new bursts of star formation, or stripping the galaxy of its gas, rendering it less able to form new stars. One important question is what effect, if any, do the intermediate density environments of cluster connecting filaments have on galaxy evolution?

Gallazzi et al. (2009) find that the starforming galaxies in the supercluster A900/A901 are mostly found in between the clusters, in regions of intermediate galaxy density. The largest sample of cluster-scale filaments has been complied in an optical survey by Porter et al. (2008) who look at $~ 50$ filaments which each connect two rich clusters. These authors also find that the mean star formation rate is greatest in galaxies a few megaparsecs from the closest cluster, that is to say, in the intermediate density filament.

Our study of Abell 1763, Abell 1770, and the $13 \mathrm{Mpc}$ long filament which connects the two clusters is based on a multiwavength analysis which spans 16 bands from the SDSS optical filters, to $1.4 \mathrm{GHz}$ radio observations. We include spectra of $\sim 1000$ sources, over 300 of which are now confirmed members (Fadda et al. 2008; Edwards et al. 2010a). This proceedings will discuss our overall main results - that there are more starforming galaxies in the filament than anywhere else in the structure. As well, we discuss the 


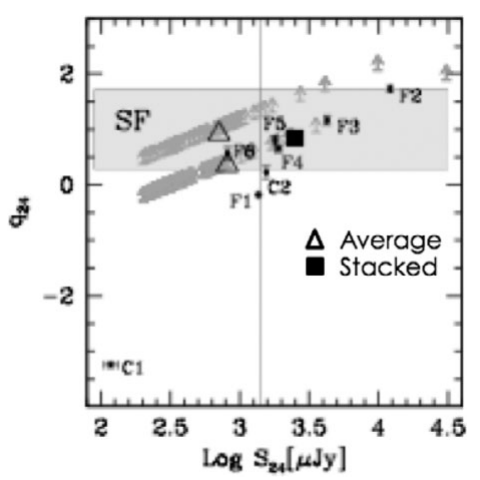

Figure 1. Galaxies with radio and mid-infrared emission are plotted on the FIR-Radio correlation. The AGN region is below the grey bar. Nearly every point is within the starforming region. The average of Radio+IR sources is within the starforming region, and the stacked value (for objects with IR emission, but no radio emission) is also well within the starforming region. The labeled galaxies correspond to those of Fig. 2.

discovery of the first bent double lobe radio source in a known cluster filament, which has allowed us to constrain the particle density of the filament medium.

\section{The filament is ripe with starforming galaxies}

As detailed in Edwards et al. 2010b, we construct spectral energy distributions for each of our mid-infrared bright galaxies using our optical, near, and mid-infrared photometry, matching the galaxy photometry to that of the best template spectra, and converting the total infrared luminosity into a star formation rate. We use the K-band luminosity to calculate the stellar mass. We find that specific star formation rates are higher for galaxies in the filament than for anywhere else in the superstructure, as well as that the frequency of star forming galaxies is highest in the filament.

Mid-infrared emission can signal the presence of an AGN as well as starformation. Therefore, to convince ourselves that the infrared-emitting galaxies are in fact starforming we perform several tests to determine the amount of AGN contamination. We use the IRAC colour-colour and optical emission line diagnostic plots, cross-match sources to quasar catalogs, and check the FIR-Radio correlation, which is shown in Fig. 1. We always find that the level of AGN contamination is very low.

The only exception is for the eight galaxies that are very bright at both radio and mid-infrared wavelengths, all of which are projected along the cluster filament, as shown in Fig. 2. Of these, three are bright AGN (including the brightest cluster galaxy) and five are star forming. For the latter, we calculate star formation rates between $10-100 \mathrm{M}_{\odot}$ per year, whether using radio fluxes, $24 \mu \mathrm{m}$ fluxes, total infrared luminosities, or the $\mathrm{H} \alpha$ emission line flux.

\section{Two bright wide angle tail galaxies give clues to cluster dynamics}

The brightest cluster galaxy (BCG) for Abell 1763 is a bright radio AGN. It is a wide angle tail galaxy, with radio lobes that are swept back. Adding to the intregue, the morphology of the cluster X-ray gas follows that of the radio lobes. The BCG is also slightly displaced from the center of the X-ray emission. All of this supports the hypothesis of streaming motions in the cluster. 


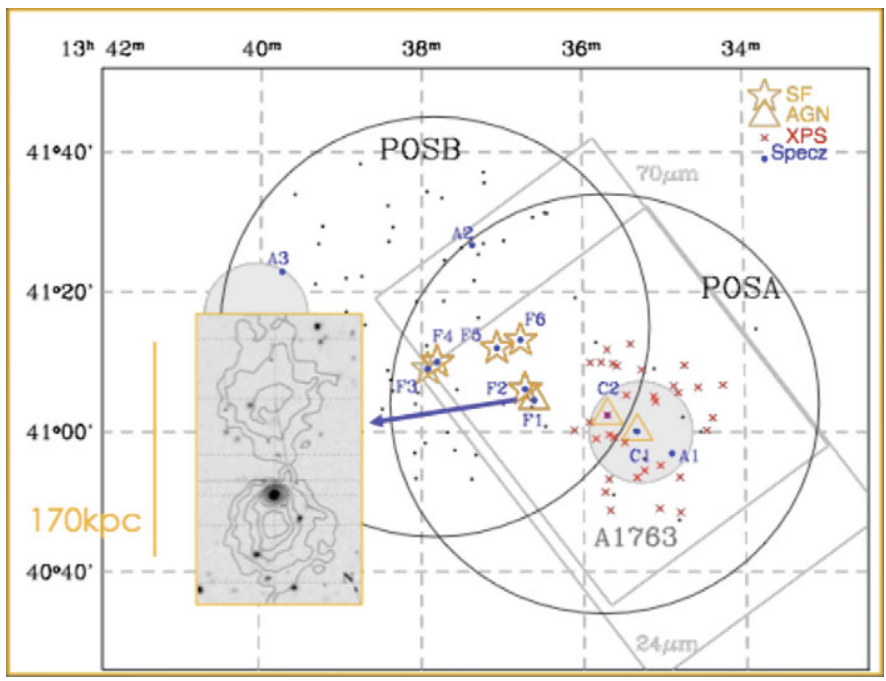

Figure 2. Abell 1763 cluster and bright galaxies along the large-scale filament. Galaxies that are very bright in both radio and mid-infrared wavelengths are labeled as stars and triangles, and all are along the direction of the filament. The radio contours of the bent double lobe radio galaxy F1 are shown in the inset on top of an optical r-band image. The two radio pointings are shown as large black circles, and the extent of the mid-infrared observations include the grey rectangles.

This central $\mathrm{cD}$ galaxy, however, is not the only bent double lobe radio source in the superstructure. There exists a second, $\sim 3.4 \mathrm{Mpc}$ from the center of Abell 1763. This second bright radio source is also projected along the filament, and its radio lobes are bent, perpendicular to the direction of the filament.

We assume that the length of the two jets is intrinsically equal. Therefore, the difference in observed jet length is purely a function of viewing angle. In Edwards et al. (2010c), we use geometric models to determine constraints on the bend, orientation and curvature radius for the jets. For reasonable values of intrinsic jet length, both the bend and orientation are tightly constrainted. We use the Euler Equation to calculate the density of the medium which pushes back on the radio lobes, causing the bend. We calculate this for several values of the spectral index, as well as a function of curvature radius, which is difficult to determine given our viewing angle. After a correction for relativistic deboosting, we arrive at $\rho_{I F M}=1-20 \times 10^{-29} \mathrm{gm} / \mathrm{cm}^{3}$ as shown in Fig. 3 .

\section{Conclusions}

The baryonic mass in superclusters is dominated by the X-ray emitting intra-cluster gas which permeates throughout clusters and the filaments between them. To date, limited observations have been conducted: One pointed, deep X-ray measurement has been published by Werner et al. (2008) of the filament medium between Abell 222/223, and Fang et al. (2010) has looked at absorption lines from background quasars by the Warm Hot Intergalactic Medium (WHIM). It has been postulated that bent double lobe radio galaxies should exist in filaments (e.g. Freeland et al. 2008), but up until now none has been verified.

Because of our a priori knowledge of the filament between Abell 1763 and Abell 1770, we are able to deduce a value for the WHIM in this filament. Our constraint of the filament density is consistent with those from numerical models (e.g. Davé et al. 2001) 


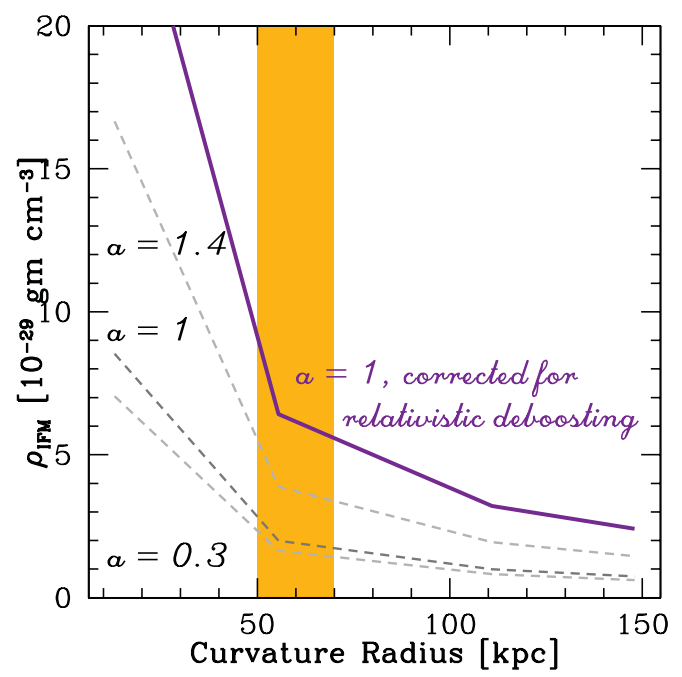

Figure 3. Constraints on the intra-filament medium for several values of curvature radius. The solid line assumes a correction for relativistic deboosting and a spectral index of 1 . The dashed lines are un-corrected for relatavistic effects and assume the spectral index labeled.

as well as the observations outlined above. Cluster feeding filaments are not just made of galaxies, but a significant medium which can bend the radio jets of an AGN. In the case of Abell 1763, the filament is the region with the most active star formation. We end asking if the filament may be able to pre-process galaxies on their way into clusters, a phenomena often associated within the environment of groups.

\section{References}

Davé, R., et al. 2001, Astrophysical Journal, 552, 473

Edwards, L. O. V., Fadda, D., Biviano, A., \& Marleau, F. R. 2010, Astronomical Journal, 139, 434

Edwards, L. O. V., Fadda, D., Frayer, D. T., Lima Neto, G. B., \& Durret, F. 2010, Astronomical Journal, 140, 1891

Edwards, L. O. V., Fadda, D., \& Frayer, D. T. 2010, Astrophysical Journal Letters, 724, 143

Fadda, D., Biviano, A., Marleau, F. R., Storrie-Lombardi, L., \& Durret, F. 2008, Astrophysical Journal Letters, 672, 9

Fang, T., et al. 2010, Astrophysical Journal, 714, 1715

Freeland, E., Cardoso, R. F., \& Wilcots, E. 2008, Astrophysical Journal, 685, 858

Gallazzi, A., et al. 2009, Astrophysical Journal, 690, 1883

Porter, S. C., Raychaudhury, S., Pimbblet, K. A., \& Drinkwater, M. J. 2008, Monthly Notices of the Royal Astronomical Society, 388, 1152

Werner, N., Finoguenov, A., Kaastra, J. S., Simionescu, A., Dietrich, J. P., Vink, J., \& Böhringer, H. 2008, Astronomy $\& 5$ Astrophysics, 482, 29 